\title{
COMPARATIVE STUDY OF THORACIC PARAVERTEBRAL BLOCK AND GENERAL ANAESTHESIA FOR POST OPERATIVE ANALGESIA IN ELECTIVE BREAST SURGERY
}

Gautam Piplai, Manas Karmakar, Dhurjoti Prasad Bhattacharya, Jatisankar Rudra, Tapan Kumar Sahoo, Sariful Alam Mallick, Arunima Mukhopadhyay, Sabyasachi Sarkar
1. Assistant Professor. Department of Anaesthesiology, Calcutta National Medical College, Kolkatta,
2. Post Graduate Trainee. Department of Anaesthesiology, Calcutta National Medical College, Kolkatta,
3. Professor. Department of Anaesthesiology, Calcutta National Medical College, Kolkatta,
4. Professor. Department of Anaesthesiology, Calcutta National Medical College, Kolkatta,
5. Post Graduate Trainee. Department of Anaesthesiology, Calcutta National Medical College, Kolkatta,
6. Post Graduate Trainee. Department of Anaesthesiology, Calcutta National Medical College, Kolkatta,
7. Assistant Professor. Department of Surgery, Calcutta National Medical College, Kolkatta,
8. Assistant Professor. Department of Surgery, Calcutta National Medical College, Kolkatta,

\section{CORRESPONDING AUTHOR:}

Dr. Gautam Piplai,

Dept. of Anaesthesiology,

Calcutta National Medical College,

Kolkata-14.

E-mail: mpiplai@yahoo.com

ABSTRACT: BACKGROUND: this randomised control trial is to evaluate unilateral paravertebral block in elective breast surgery in comparison with general anaesthesia METHODS: 106 patients of breast surgery were randomised in 2 groups- group-P and group-G. group-P patients received unilateral multiple level paravertebral block with $0.25 \%$ levo-bupivacaine at $\mathrm{T} 2$ to $\mathrm{T} 5$ level and group-G patients received general anaesthesia. Pulse, BP were recorded in the perioperative period and rescue analgesic requirement time and VAS scores were recorded in PACU. Recorded data were evaluated after that. RESULTS: In PACU rescue analgesia needed in group-P in 69.22 minutes and in group-G in 41.27 minutes. Post-operative VAS scores were lower in group-P patients at 30 minutes, $1 \mathrm{hr}$. and $2 \mathrm{hr}$. There was no incidence of hypotension or hypertension or bradycardia or tachycardia in any group. Patients overall satisfaction score in group-P was 4 vs 3.6 in group-G (p value $<0.05$ ) CONCLUSION: Thoracic para-vertebral block is a safe alternative to general anaesthesia for elective breast surgery as it provides better postoperative analgesia with similar hemodynamic stability.

KEY WORDS: paravertebral block, general anaesthesia, breast surgery, post operative analgesia, levobupivacaine

INTRODUCTION: General anaesthesia is age old technique for elective breast surgery. Recently other modes of anaesthesia/ analgesia like thoracic epidural or thoracic paravertebral block can also be applied for that purpose. The advantages of these techniques minimally hinders airway, prevents use of muscle relaxants, and decreases chance of aspiration, post-operative nausea, and vomiting. Moreover general anaesthesia alone does not help much in post-operative analgesia.

Para-vertebral block involves injection of local anaesthetic in a space immediately lateral to where the spinal nerves emerge from the inter-vertebral foramina. This technique is being used increasingly as a sole anaesthetic technique for carrying out various procedures like 
thoracotomy, breast surgery and chest wall trauma management. This popularity is mainly due to the ease of the technique and fewer complications.

MATERIALS \& METHODS: After obtaining approval from the institution ethical committee, comparison of hemodynamic status and postoperative analgesic effect of paravertebral block and general anaesthesia in elective breast surgery was done. All elective breast surgery cases starting from $1^{\text {st }}$ June to September 30, 2012 [4 months] age between 18-65 with ASA physical status I \& II were enrolled for the study. Written and informed consent was taken from all patients. Patients having any condition contraindicating elective surgery or fit in exclusion criteria (list of our exclusion criteria given below), then she was excluded from study.

All patients were randomised by a computer generated random number table into two groups, namely group-G (for general anaesthesia) and group-P (for para-vertebral block).All patients were counselled regarding anaesthetic procedure in the pre-anaesthetic examination room on the day before operation. All of them got $10 \mathrm{mg}$ of oral diazepam before going to bed on the night before surgery and in the early morning on the day of surgery.

On arrival to Operating Room, in all patients iv access was established and multipara monitors were attached and baseline recording were recorded. In group-G patients were preoxygenated, received inj. Fentanyl at $2 \mathrm{mcg} / \mathrm{kg}$ body wt. 3 minutes prior to induction. They were induced with inj. propofol $2 \mathrm{mg} / \mathrm{kg}$ body wt in incremental doses until the verbal response was lost. They were intubated under succinylcholine $1.5 \mathrm{mg} / \mathrm{kg}$ body wt. and maintained with infusion propofol at $50 \mathrm{mcg} / \mathrm{kg} \mathrm{b}$. wt./min, 33\% 02 in N2O. After recovery from depolaring block as seen clinically and TOF response, a loading dose of $0.5 \mathrm{mg} / \mathrm{kg}$ body wt. Atracurium besylate given intravenously and repeat dose of $5 \mathrm{mg}$ atracurium given each time by observing TOF response more or equal to 2 out of 4 . Repeat doses of fentanyl [ $0.5 \mathrm{mcg} / \mathrm{kg}$ body wt.] given every 30 minutes. The dose of infusion propofol was adjusted to keep the BIS score between 40 to 60. Any episode of hypotension will be treated with increased amount of intravenous fluids and vasopressors, if needed.

In group P patients, they were positioned laterally according to diseased side which was kept up. After appropriate paint and draping, sites for para-vertebral injection were identified, marked and infiltrated with $1 \mathrm{ml}$ lignocaine $2 \%$ in each space.Then they received unilateral multiple level para-vertebral block at T2 to T5 level using loss of resistance technique (described below) using 18G Tuohy needle. $5 \mathrm{ml}$ of $0.25 \%$ levo-bupivacaine was injected in each para-vertebral space. Then they were sedated with intravenous propofol infusion at $50 \mathrm{mcg} / \mathrm{kg}$ body wt./min. Rate of propofol infusion titrated to keep BIS score 60 to 80. Sternal border of diseased breast locally infiltrated with $5 \mathrm{ml} 0.25 \%$ levo-bupivacaine. If surgical anaesthesia was not achieved within 20 minutes, then they were given general anaesthesia and excluded from further evaluation. Intraoperatively if the heart rate was elevated by $20 \%$ then inj. Fentanyl 0.5 $\mathrm{mcg} / \mathrm{kg}$ body wt was given.

Pulse rate, Heart rate, NIBP, Sp02, BIS score were recorded every 5 minutes. Duration of surgery and duration of Operating Room use were also noted in every case. After adequate reversal, all patients were taken to PACU to monitor next. VAS score checked every ten minutes. Inj. Diclofenac sodium $1 \mathrm{mg} / \mathrm{kg}$ IM was given as rescue analgesic, when patient asked for it or VAS is $\geq 4$. At the end of procedure patients were asked for their over all satisfaction and a score was allotted according to that (1-not satisfied to 5-very much satisfied)

All data were tested by independent Student's t-test, or chi-square test or ANOVA as appropriate. $P$ value $<0.05$ is taken as significant. 
Exclusion criteria: Inability to provide informed consent

Pregnancy or breast-feeding mothers

Severe obesity (body mass index $>35 \mathrm{~kg} / \mathrm{m} 2$ )

Coagulation disorders or treatment with anticoagulants

Allergy to local anaesthetics

Severe spine or chest wall deformity

Infection at the thoracic injection site

Surgical complications requiring re-operation

$\mathrm{H} / \mathrm{o}$ cardiac ischaemia or valvular heart disease

ASA physical status III and IV

Technique of para-vertebral block (using loss of resistance technique) Patient positioned in the lateral position with the neck flexed, back arched, and hip and knee flexed. Spines of appropriate level are identified and marked. A point $2.5 \mathrm{~cm}$ lateral to the spine marked with skin marker. Following strict aseptic precautions the site of injection is infiltrated with $2 \%$ lignocaine $1 \mathrm{ml}$ in each site. 18G Tuohy needle is introduced through the mark and advanced directly perpendicular to the skin, until contact with transverse process of the particular vertebra. Loss-of-resistance syringe is attached to the needle and while continuously testing for loss of resistance to saline the needle is "walked off" the structure and advanced in infero-lateral direction gradually ensuring that the bevel of the needle points laterally. As the costo-transverse ligament is penetrated, a "pop" is felt, and there is a loss of resistance to saline. This signifies para-vertebral space and drug is injected here.

RESULTS: Out of 213 patients who underwent breast surgery during that period 116 patients consented for the study. They were randomised in two groups --- 58 in each group. 3 patients of group-G were excluded from study due to profuse surgical blood loss. 7 patients of group-P were excluded from the study as 3 out of 7 withdrew consent at the last moment, 2 out of 7 suffered from severe surgical blood loss, and in 2 out of 7 were switched over to general anesthesia as paravertebral block failed to achieve surgical analgesia. Patients who suffered severe surgical blood loss were treated with intravenous fluids, blood and if needed vasopressor. So altogether 55 patients were included in group-G and 51 patients were included in group-P.

Average weights, height of patients in both groups were comparable with each other with a p-value $>0.05$ [table1]. Average duration of surgery were similar in both groups (118.6 minutes in group-P vs 121.3 minutes in group-G with p-value $>0.05$ ).

However the average duration of Operating Room usage was higher in group-P (147.7 minutes in group-P vs 128.4 minutes in group-G with p-value $<0.05$ ).

Pulse, heart rate and blood pressure in both groups were comparable when analysed with ANOVA. Baseline pulse, heart rate and blood pressures were also comparable between two groups. No incidence of significant hypertension or hypotension, bradycardia or tachycardia noticed in any patient of any group after exclusion of those stated above. So it can be concluded that patients in both groups were similarly hemodynamically stable. 
Average time of rescue analgesia (from arrival in PACU to receiving first dose of rescue analgesia) in group-P was 69.22 minutes and in group-G was 41.27 minutes with a p value of $<0.001$ which is highly significant.

VAS scores of both groups were analysed by ANOVA and VAS scores of group-P were significantly lower at 30 minutes, 1 hour, and 2hours after admission into PACU. However VAS score at 0 minutes, 4 hours, 8 hours and 24 hours were similar in both groups. In the first 24 hours period after surgery, average requirement amount of Diclofenac sodium was 216.2 $\mathrm{mg} /$ patient in group-P and $229.1 \mathrm{mg} /$ patient in group-G. The difference was significant with a $p$-value $<0.05$. So patients of group-P had better post-operative analgesia than group-G. Patients of group-P had an average overall satisfaction score of 4 and group-G 3.6 and this was also significant ( $p$ value $<0.05)$.

DISCUSSION: Thoracic para-vertebral block is a safe alternative to general anaesthesia for elective breast surgery as it provides better postoperative analgesia with similar hemodynamic stability.

Hugo Sellheim of Leipzig was first to perform a para-vertebral block to provide analgesia and muscle relaxation avoiding spinal anaesthesia. His resident Lawen studied the procedure and developed it as a diagnostic tool for abdominal pain. In 1920s it became highly popular and then gradually phased out from common practice. In 1970s publications again started to reappear regarding its use and gradually it has become popular now because of technical upgradation and more skilful approach with fewer complications.

Eamon Coveney et al [1] in 1998 in a case series of 156 patients showed that paravertebral block can be used to perform major operations for breast cancer with minimal complications and a low rate of conversion to general anesthesia. Paravertebral block markedly improves the quality of recovery after breast cancer surgery and provides the patient with the option of ambulatory discharge.

Stephen M. Klein et al [2] in a single-blinded, prospective randomized study of 60 women scheduled for unilateral or bilateral breast augmentation or reconstruction concluded that PVB is an alternative technique for cosmetic breast surgery that may offer superior pain relief and decreased nausea alone.

In 2006 Pekka M. Kairaluoma et al [3] followed up 60 patients of paravertebral block upto $1 \mathrm{yr}$ after breast surgery and found that the incidence of neuropathic pain was low (two and three patients in the PVB and control groups, respectively). In addition to providing acute postoperative pain relief, preoperative PVB seems to reduce the prevalence of chronic pain $1 \mathrm{yr}$ after breast cancer surgery.

Jytte F. Moller et al [4] in 2007 in a double-blinded randomized controlled trial of 79 patients showed that a multilevel paravertebral block provides good analgesia for breast surgery.

A. Schnabel et al [5] did a meta-analysis of fifteen randomized controlled trials (published between 1999 and 2009) including 877 patients and that showed that there is considerable evidence that PVB in addition to general anaesthesia or alone provide a better postoperative pain control with little adverse effects compared with other analgesic treatment strategies.

In 2012 S.Das et al [6] published a randomised control trial in Indian journal of anaesthesia that unilateral paravertebral block was more efficacious than general anaesthesia in prolonging post operative analgesia and reducing morbidities in elective breast surgery. 
We have also found better post-operative analgesia with unilateral multiple level paravertebral block in breast surgeries than general anaesthesia alone. Parasternal subcutaneous infiltration of diseased breast side with $5 \mathrm{ml}$. Levobupivacaine( $0.25 \%$ ) reduced need for general anaesthesia conversion to $0 \%$.

\section{Anatomy of para-vertebral space:}

wedge-shaped potential space just lateral to vertebra bounded by

antero-laterally: by parietal pleura

posteriorly : by superior costo transverse ligament

superiorly and inferiorly: by the heads of the ribs

medially: by body of vertebra, intervertebral disc and intervertebral foramina

contents: the spinal root emerges from the intervertebral foramen and divides into dorsal and ventral rami. The sympathetic chain lies in the same fascial plane, just anterior to the intercostal nerve and communicates with it via the rami communicants

The space is continuous with the intercostal space laterally, paravertebral space above and below, the epidural space medially and the contralateral paravertebral space through the paravertebral and epidural space.

\section{REFERENCES:}

1. Eamonn Coveney, MB, FRCSI, Christina R. Weltz, MD, Roy Greengrass, MD,t J. Dirk Iglehart, MD, George S. Leight, MD, Use of Paravertebral Block Anesthesia in the Surgical Management of Breast Cancer ANNALS OF SURGERY Vol. 227, No. 4, 496-501

2. Thoracic Paravertebral Block for Breast Surgery ; Stephen M. Klein, MD, Arthur Bergh, MD, PhD, Susan M. Steele, MD, Gregory S. Georgiade, MD, and Roy A. Greengrass, MD, FRCP ; AnesthAnalg 2000;90:1402-5

3. Preincisional Paravertebral Block Reduces the Prevalence of Chronic Pain After Breast Surgery ;Pekka M. Kairaluoma, MD Martina S. Bachmann, MD, PhD Per H. Rosenberg, MD, PhD Pertti J. Pere, MD, PhD ; AnesthAnalg 2006;103:703-8

4. Thoracic Paravertebral Block for Breast Cancer Surgery: A Randomized Double-Blind Study Jytte F. Moller, MD, PhD Lone Nikolajsen, MD, PhD SveinAageRodt, MD, PhD Hanne Ronning, MD Palle S. Carlsson, MD, PhD ;AnesthAnalg 2007;105:1848 -51

5. Efficacy and safety of paravertebral blocks in breast surgery: a meta-analysis of randomized controlled trials A. Schnabel, S. U. Reichl, P. Kranke, E. M. Pogatzki-Zahn and P. K. Zahn ; British Journal of Anaesthesia Page 1 of 11 doi:10.1093/bja/aeq265

6. Multiple-injection thoracic paravertebral block as an alternative to general anaesthesia for elective breast surgeries: A randomised controlled trial ;Sabyasachi Das, Pradipta Bhattacharya, Mohan Chandra Mandal, Soma Mukhopadhyay, SekharRanjanBasu, Bikas Kusum Mandal ; IndianJAnaesth56127-2894546_080225 
Demographic profile

\begin{tabular}{|c|c|c|}
\hline & Weight & Height \\
\hline & In kg & In cm \\
\hline p value & $>0.05$ & $>0.05$ \\
\hline Group--P & 50.3 & 157.3 \\
\hline Group-G & 52.5 & 159.1 \\
\hline
\end{tabular}
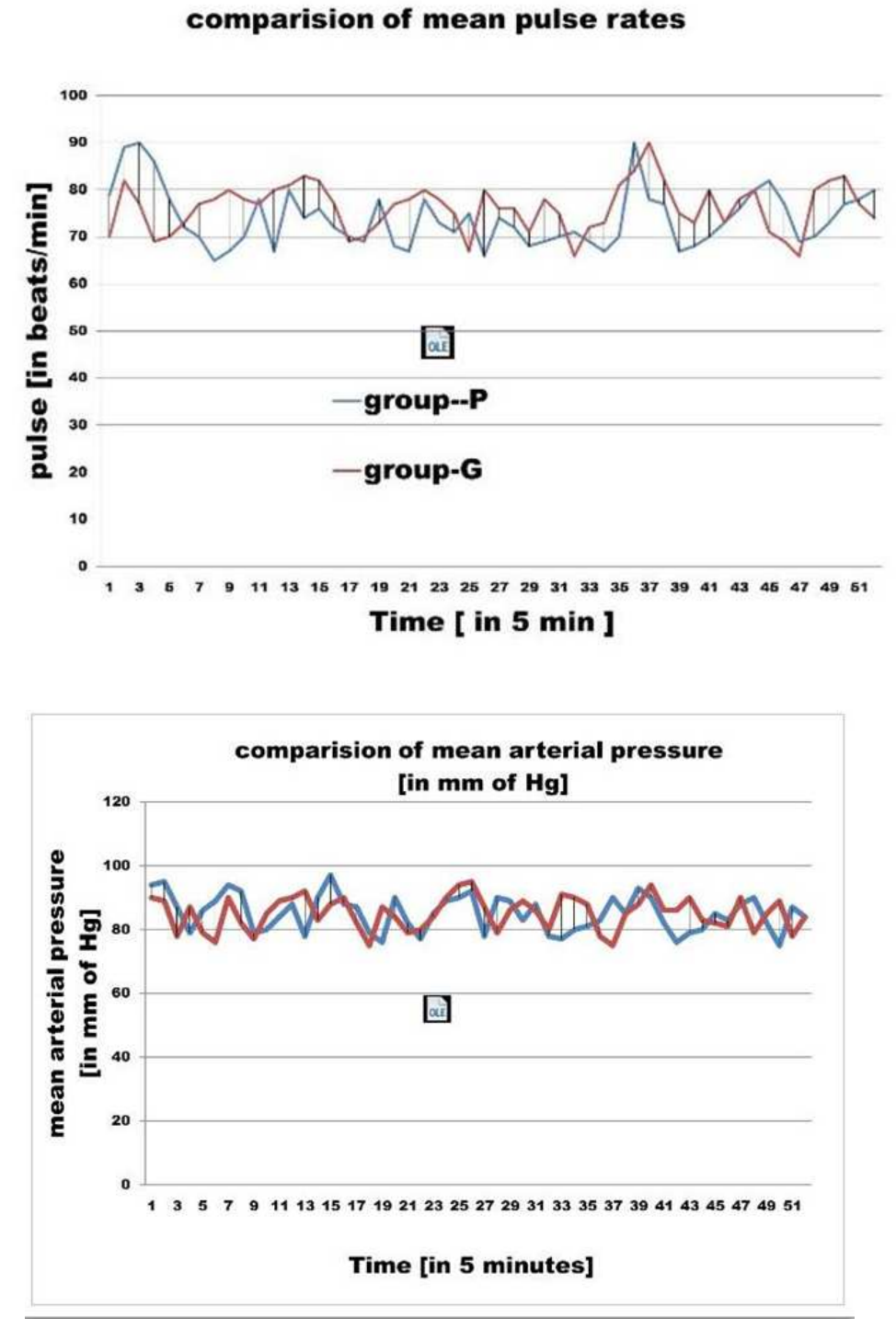

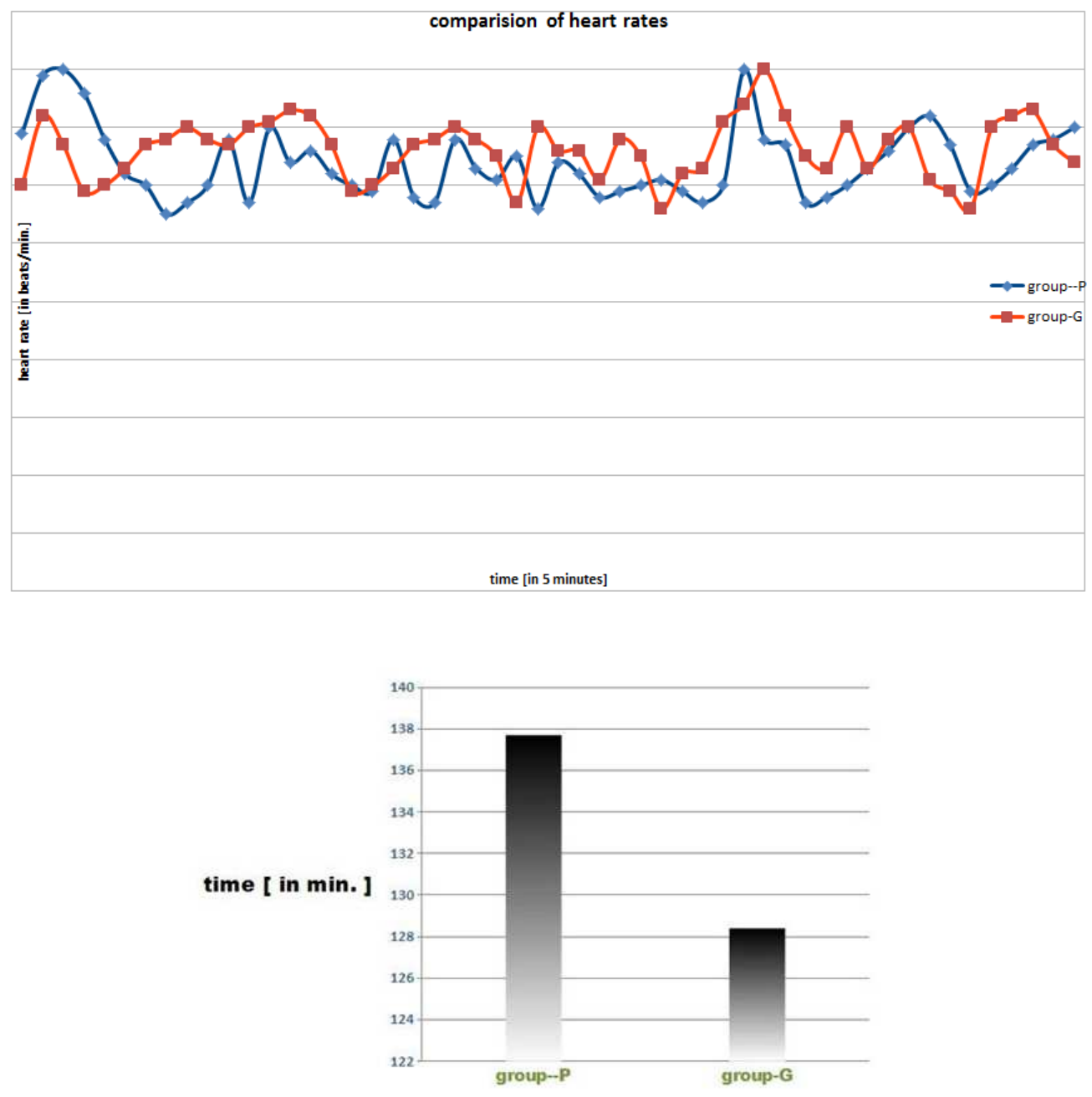

duration of OR use

duration of surgery

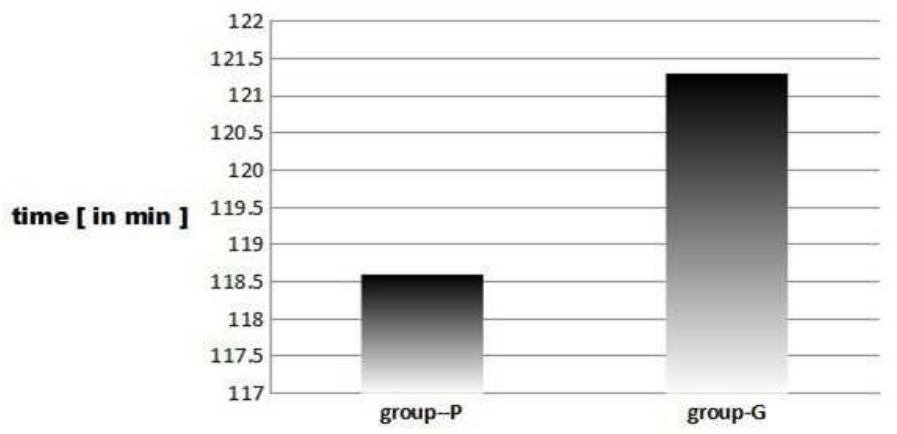


average duration before of rescue analgesia
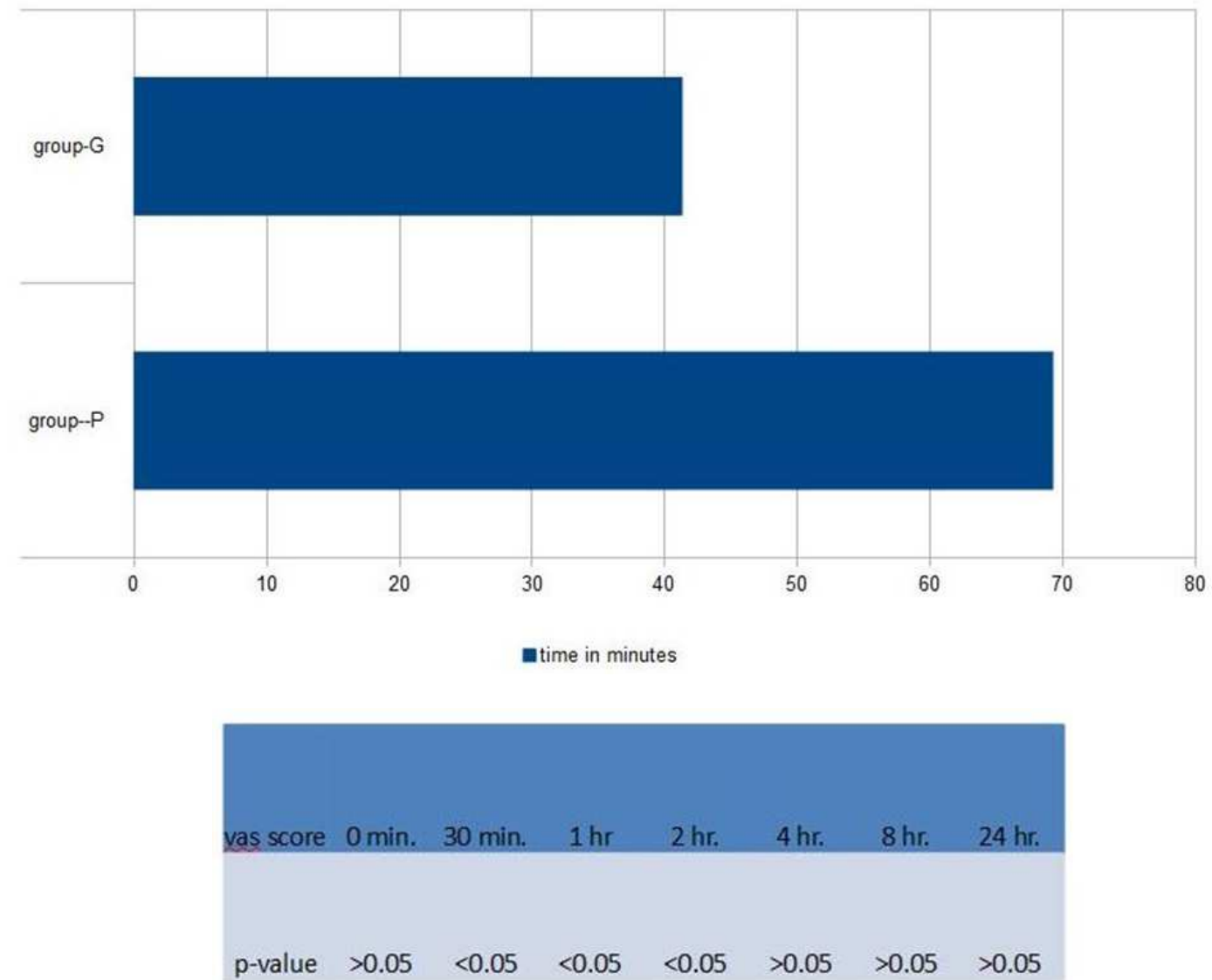

$\begin{array}{llllllll}\text { group--P } & 2.1 & 2.8 & 3.3 & 4.2 & 4.3 & 4.1 & 3.9\end{array}$

$\begin{array}{llllllll}\text { group-G } & 2.3 & 3.4 & 4 & 4.7 & 4.8 & 4.2 & 3.9\end{array}$

VAS SCORES

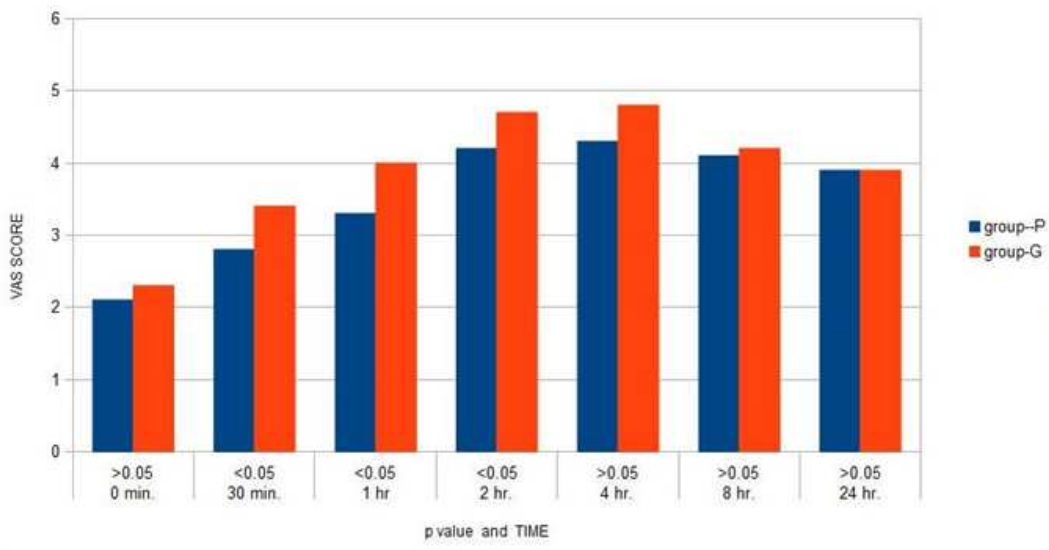




\section{diclofenac requirred in 24 hrs. [in $\mathbf{~ m g ]}$}

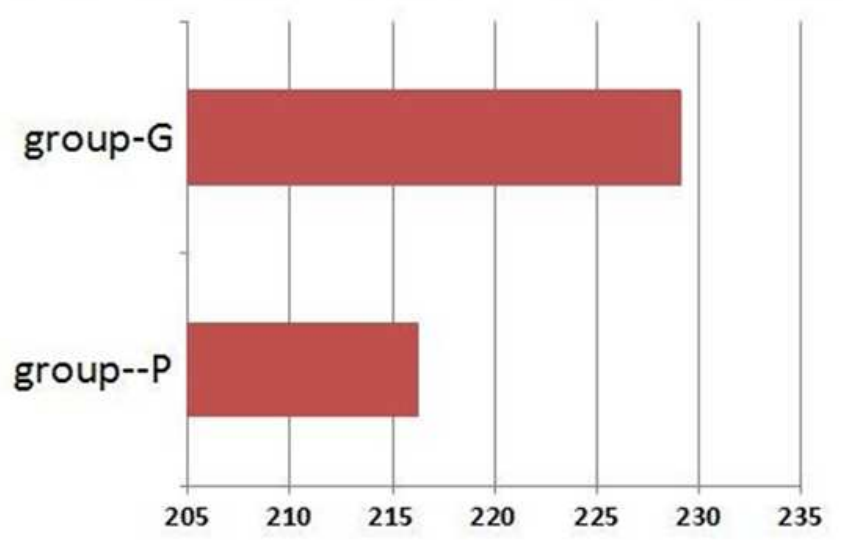

over all satisfaction score

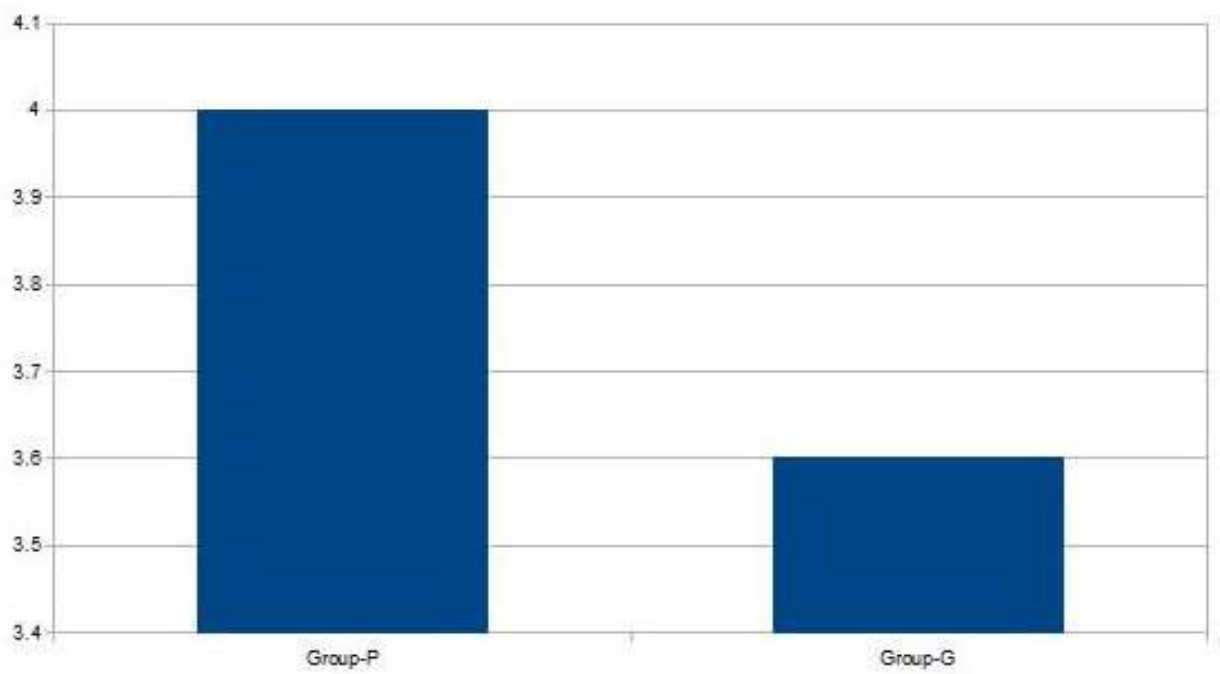

